\title{
Iniciação esportiva para escolares: os impactos na coordenação e no desempenho motor após um programa de ensino
}

\author{
Sports initiation for schoolchildren: impacts on \\ coordination and motor performance after a teaching \\ program
}

Iniciación deportiva para estudiantes: los impactos en la coordinación y en el desempeño motriz después de un programa de enseñanza

\footnotetext{
iD Nayanne Dias Araújo

Universidade Federal de Mato Grosso, Cuiabá, Mato Grosso, Brasil. nayanne_araujo@hotmail.com

(iD) Henrique de Oliveira Castro Universidade Federal de Mato Grosso, Cuiabá, Mato Grosso, Brasil. henriquecastro88@yahoo.com.br

iD A Allana Lendary Bernardo Ramos Universidade Federal de Mato Grosso, Cuiabá, Mato Grosso, Brasil. allana_lendary@hotmail.com

iD (9) Tatiane Mazzardo Universidade Federal de Minas Gerais, Belo Horizonte, Minas Gerais, Brasil. tatimazzardo@hotmail.com

iD (9) Gabriella Nelli Monteiro Faculdade Centro Mato-Gossense, Sorriso, Mato Grosso, Brasil. gnm_cba@hotnail.com

iD Layla Maria Campos Aburachid Universidade Federal de Mato Grosso, Cuiabá, Mato Grosso, Brasil. laylabur@hotmail.com
} 
Resumo: O objetivo do estudo foi identificar os impactos das propostas metodológicas, Iniciação Esportiva Universal e Escola da Bola, sobre o nível de coordenação motora e desenvolvimento motor de alunos participantes de aulas de Educação Física escolar. Participaram 40 crianças (grupo experimental e controle) com idade entre oito e 10 anos $(7,85 \pm 0,48)$, de ambos os sexos. Durante 17 sessões, os sujeitos vivenciaram os conteúdos inerentes às propostas metodológicas, que tiveram sua concordância e coerência comprovados pela categorização das aulas. A aprendizagem incidental foi capaz de provocar melhorias na coordenação motora e no desenvolvimento motor, com grande efeito após a intervenção e aumento de chances de alteração das classificações propostas pelos testes aplicados.

Palavras-chave: Crianças. Educação Física. Aprendizagem.

\begin{abstract}
Theaim ofstudywasto identifytheimpacts ofthemethodological proposals Iniciação Esportiva Universal and Ball School on the level of motor coordination and motor performance of students participating in school Physical Education classes. 40 children participated (experimental and control group) aged 8 to 10 years $(7.85 \pm 0.48)$, of both genders. During 17 sessions, the subjects experienced the contents inherent to the methodological proposals, with agreement and coherence proven by class categorization. Incidental learning was able to cause improvements in motor coordination and motor performance, with large effect size after intervention and increased chances of changing the classifications proposed by the applied tests.
\end{abstract}

Keywords: Children. Physical Education. Learning.

Resumen: El objetivo del estudio ha sido identificar los impactos de las propuestas metodológicas de Iniciación Deportiva Universal y Escuela del Balón sobre el nivel de coordinación motriz y motricidad de alumnos practicantes de clases de Educación Física escolar. 40 niños y niñas han participado (grupo experimental y control) con edad entre 8 y 10 años $(7,85 \pm 0,48)$. Durante 17 sesiones, experimentaron los contenidos inherentes a las propuestas metodológicas, que tuvieron su consistencia 
y coherencia comprobados por la categorización de los contenidos desarrollados en las aulas. El aprendizaje incidental provocó mejoras en la coordinación motriz y motricidad, con tamaño del efecto grande después de la intervención y aumento de posibilidades de cambio de las clasificaciones propuestas por los testes aplicados.

Palabras-clave: Niños. Educación Física. Aprendizaje.

Submetido em: 30-08-2021

Aceito em: 09-09-2021 
Iniciação esportiva para escolares: os impactos na coordenação e no desempenho motor...

Nayanne Dias Araújo - Henrique de Oliveira Castro - Allana Lendary Bernardo Ramos - Tatiane Mazzardo • Gabriella Nelli Monteiro • Layla Maria Campos Aburachid

\section{Introdução}

As transformações, ocorridas nos últimos anos, na pedagogia aplicada à Educação Física no contexto escolar proporcionam aos professores direcionamentos para o que ensinar e para o que os alunos devem aprender. Antes do planejamento e implementação, por exemplo, de um método de ensino, os professores devem considerar várias questões que orientam não só o processo de seleção de suas atividades, mas, como afirmam Gurvitch e Metzler (2013), devem considerar ainda as competências (tipos de resultados de aprendizagem pretendidos, enquadramento da tarefa de cada atividade de aprendizagem e envolvimento dos alunos) para além do conhecimento do conteúdo.

As formas de iniciar a criança no esporte, visando sua formação geral, receberam novas estruturações e reformulações, chegando a se tornarem propostas metodológicas de ensino comprovadas empiricamente por meio de estudos de intervenção (GRECO; MEMMERT; MORALES, 2010; MEMMERT, 2006; MEMMERT; ROTH, 2007; MESQUITA et al., 2005). Estas propostas de iniciação esportiva se contrapõem aos métodos nomeados como tradicionais (analítico, global e misto), sendo anunciadas como métodos contemporâneos de ensino. Elas trazem consigo características pedagógicas comuns que geralmente contemplam os seguintes pontos: 0 aluno é o centro do processo de ensino, o domínio da prática é geral, o jogo deve ser estimulado desde o início, e a aprendizagem incidental deve ser estimulada antes da aprendizagem intencional. Diz-se, geralmente, que as intensidades de estímulos por aprendizagem intencional e incidental provocam variação no domínio de prática, como no caso do Teaching Games for Unserstanding (TGfU) e do Modelo Desenvolvimentista (ABURACHID et al., 2019). No Brasil, a comunidade acadêmica passou a se preocupar com as diferentes maneiras de desenvolver o processo de ensino-aprendizagem de crianças, com a realização de estudos sobre a iniciação esportiva, principalmente nos últimos quatro anos (ABURACHID et al., 2019; 
Iniciação esportiva para escolares: os impactos na coordenação e no desempenho motor...

Nayanne Dias Araújo - Henrique de Oliveira Castro - Allana Lendary Bernardo Ramos - Tatiane Mazzardo • Gabriella Nelli Monteiro • Layla Maria Campos Aburachid

BETTEGA et al., 2019; KRAHENBÜHL; LEONARDO, 2018; LAGES et al., 2021; VAZ et al., 2021).

Kleynen e colaboradores (2014) conceituam a aprendizagem intencional/explícita como uma aprendizagem que gera conhecimento verbal no desempenho do movimento, e necessita do envolvimento da memória de trabalho. Por outro lado, a aprendizagem incidental/implícita envolve a aquisição não intencional e automática de conhecimento, com pouco ou quase nenhum aumento do conhecimento verbal no desempenho do movimento. Como exemplo prático de aprendizagem incidental, durante uma aula, o professor propõe aos alunos a realização de uma atividade (podendo ser um exercício ou jogo), e os alunos, individual ou coletivamente, realizam a atividade sem demais instruções específicas. Desta forma, identificam, por meio da prática, a melhor forma de executar as ações para atingir seu objetivo. A Iniciação Esportiva Universal (IEU) (GRECO; BENDA, 1998) e a Escola da Bola (EB) (KRÖGER; ROTH, 2005) são propostas metodológicas contemporâneas que estimulam a aprendizagem incidental e o domínio geral da prática.

O domínio da prática decorre por meio da aprendizagem de conteúdos específicos ou gerais (RAAB, 2015). No domínio da prática específico, o professor estimula o aprendizado do aluno dirigindo-o à execução de fundamentos específicos de um esporte para chegar à melhor decisão em situações mais estreitas. Já no domínio da prática geral, os jogos táticos contemplam diferentes situações, ampliando os conceitos semelhantes entre os esportes, proporcionando, portanto, a transferência de seus conhecimentos (RAAB, 2015). As propostas metodológicas IEU e EB compreendem que a execução das tarefas para o desenvolvimento da capacidade de jogo se constrói por meio de processos pedagógicos do conhecido para o novo, do geral para o específico e do simples para o complexo (GRECO et al., 2020).

Uma das variáveis, objeto de estudos atualmente em crianças, que se utiliza das propostas da IEU e EB é o desempenho motor. O estudo de Vaz e colaboradores (2021) demonstrou melhorias 
Iniciação esportiva para escolares: os impactos na coordenação e no desempenho motor...

Nayanne Dias Araújo - Henrique de Oliveira Castro - Allana Lendary Bernardo Ramos - Tatiane Mazzardo • Gabriella Nelli Monteiro • Layla Maria Campos Aburachid

na coordenação motora grossa de 73 crianças entre entre cinco e 10 anos de idade após uma intervenção baseada na estrutura TARGET e EB. Em outro estudo, desenvolvido por Lages e colaboradores (2021), após uma intervenção com o IEU com 24 crianças de 10 a 12 anos de idade, pertencentes a escolas das áreas rural e urbana, houve melhorias na coordenação motora com bola. Adicionalmente, estudos como os realizados por Cabral, Aburachid e Greco (2012), Aburachid e colaboradores (2015), Aburachid e colaboradores (2019) e Mazzardo e colaboradores (2020), além de aplicarem conteúdos por meio da aprendizagem incidental, também os combinaram com o ensino de técnicas esportivas específicas (handebol, voleibol, futsal e badminton) por meio da aprendizagem intencional.

Conforme observado, estudos de intervenção utilizando propostas de aprendizagem incidental/implícita foram capazes de promover mudanças positivas nas variáveis de coordenação motora e desenvolvimento motor. Porém, nenhum estudo anterior avaliou os níveis de coordenação e desenvolvimento motor após uma intervenção metodológica híbrida do IEU+EB com crianças de oito a 10 anos no contexto escolar. Desta forma, o presente estudo objetiva identificar os impactos do IEU+EB para a formação esportiva geral de crianças de oito a 10 anos, no contexto de ensino escolar, sobre o nível de coordenação motora e do desenvolvimento motor, considerando os efeitos tempo e sexo, e analisar a chance de alteração na classificação das variáveis. De acordo com os estudos expostos anteriormente, formula-se a hipótese que a proposta IEU+EB promoverá melhorias na coordenação motora e no desenvolvimento motor das crianças.

\section{Materiais e Métodos}

\section{Participantes}

Inicialmente 40 escolares do ensino fundamental (grupo experimental = 20; grupo controle $=20$ ), com idade entre oito e 10 anos 
Iniciação esportiva para escolares: os impactos na coordenação e no desempenho motor...

Nayanne Dias Araújo - Henrique de Oliveira Castro - Allana Lendary Bernardo Ramos - Tatiane Mazzardo • Gabriella Nelli Monteiro • Layla Maria Campos Aburachid

$(7,85 \pm 0,48)$, determinados de forma não probabilística intencional, participariam do estudo.

Como critério de inclusão, foram selecionados os escolares que estavam na faixa etária determinada e que não praticavam atividades esportivas extracurriculares. Foram excluídos do estudo os escolares que faltaram no momento de aplicação dos testes, que faltaram mais de três aulas ou que se recusaram a participar. Uma criança do grupo controle mudou-se de escola, e por isso, foi excluída do estudo. Outro participante do grupo experimental faltou a aplicação do pós-teste. Os dados obtidos deste sujeito no pré-teste foram duplicados no pós-teste, como permite o procedimento de análise de intenção de tratar (GUPTA, 2011). Então, para o grupo controle foram analisadas 19 crianças.

\section{Instrumentos}

Para avaliação da coordenação motora, utilizou-se o Korperkoordination Test fur Kinder (KTK) (KIPHARD; SCHILLING, 1974), validado para a população brasileira por Moreira e colaboradores (2019). Para avaliação do desenvolvimento motor, utilizou-se o Test of Gross Motor Development - second edition (TGMD-2) (ULRICH, 2000), validado para a população brasileira por Valentini e colaboradores (2008).

\section{Procedimentos}

O estudo caracteriza-se como descritivo com delineamento quase-experimental de grupo controle não equivalente. $O$ estudo respeitou as normas estabelecidas pelo CNS e foi aprovado pelo CEP-UFMT sob o protocolo CAAE: 57863616.4 .0000 .5541 (parecer $n^{\circ} 1.928 .736$ ). Todos os alunos pertencentes às duas turmas do $3^{\circ}$ ano do ensino fundamental de uma escola municipal da cidade de Cuiabá-MT foram convidados a participar. O delineamento seguiu a seguinte cronologia: 
Iniciação esportiva para escolares: os impactos na coordenação e no desempenho motor...

Nayanne Dias Araújo - Henrique de Oliveira Castro - Allana Lendary Bernardo Ramos - Tatiane Mazzardo • Gabriella Nelli Monteiro • Layla Maria Campos Aburachid

a. Todos os participantes realizaram o pré-teste, a fim de quantificá-los quanto às variáveis dependentes antes da intervenção pedagógica. Neste momento foram aplicados o questionário de dados demográficos e os testes KTK e TGMD-2.

b. Durante os três meses de intervenção pedagógica, os participantes do grupo experimental vivenciaram os conteúdos inerentes às propostas metodológicas IEU+EB. Conforme Greco e colaboradores (2015) e Greco e colaboradores (2020), essas propostas se complementam quanto aos conteúdos, e, por este motivo, decidiu-se aplicá-las em conjunto. A aplicação da intervenção foi realizada pela pesquisadora responsável, a fim de garantir que os conteúdos fossem empregados conforme as propostas metodológicas e que a condição de aprendizagem emergida por parte dos alunos fosse incidental, como realizado em estudos prévios (PÍFFERO; VALENTINI, 2010; MAZZARDO et al., 2020). O grupo controle continuou recebendo aulas de Educação Física com o professor da escola. Todas as sessões de aula foram filmadas e categorizadas, conforme a planilha de categorização validada por Soares e colaboradores (2010), permitindo-se observar a qualidade intencional do professor quanto as características pedagógicas das propostas metodológicas (ABURACHID et al., 2019). No que se refere à quantidade de sessões de aulas, baseando-se em constatações de outros estudos (BRAUNER; VALENTINI, 2009; PÍFFERO; VALENTINI, 2010; MOREIRA; MATIAS; GRECO, 2013), o grupo experimental realizou 17 sessões de aulas, com duração média de 90 minutos, para verificar se a intervenção produziria resultados positivos no processo de ensino-aprendizagem. O grupo controle participou da mesma quantidade de aulas com mesmo tempo médio de duração.

c. Após o período de três meses, ambos os grupos realizaram o pós-teste. Neste momento foram aplicados os testes KTK e TGMD-2. 
Iniciação esportiva para escolares: os impactos na coordenação e no desempenho motor...

Nayanne Dias Araújo - Henrique de Oliveira Castro - Allana Lendary Bernardo Ramos - Tatiane

Mazzardo • Gabriella Nelli Monteiro • Layla Maria Campos Aburachid

Os procedimentos adotados estão descritos, de forma esquemática, na Figura 1.

Figura 1. Design experimental.

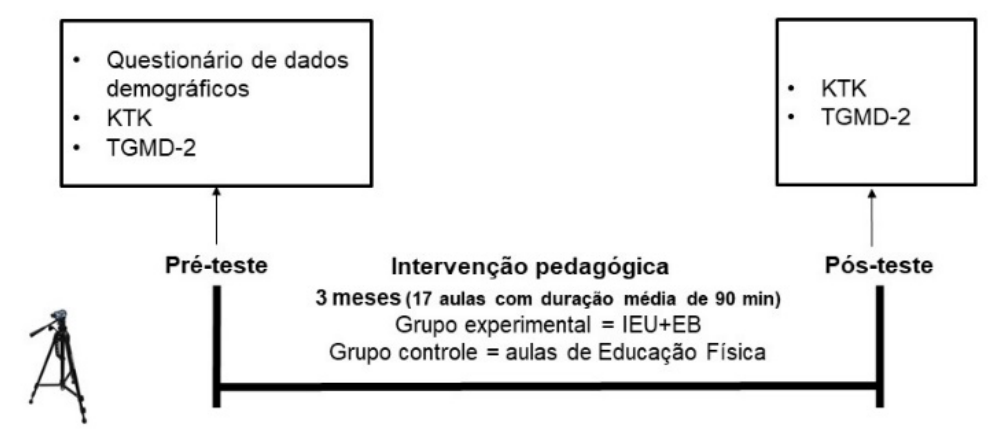

Nota: $K T K=$ Korperkoordination Test fur Kinder; TGMD-2 = Test of Gross Motor Development - second edition; IEU = Iniciação Esportiva Universal; $E B=$ Escola da bola; $\min =$ minutos.

Fonte: autores.

\section{Intervenção pedagógica}

Os participantes do grupo experimental receberam uma unidade didática conforme a proposta metodológica da IEU+EB (GRECO et al., 2015; GRECO et al., 2020), completando um tempo total de 1.524,58 minutos. A progressão pedagógica das aulas levou em consideração a estimulação diversificada dos membros do corpo, uso de distintos implementos. Elegeu-se o modelo hélice de articulação vertical e horizontal (VICKERS, 1990; RINK, 2010) para estabelecer tais progressões. Na articulação vertical, adicionam-se elementos à tarefa com níveis de dificuldade distintas, para garantir que se atinja o resultado final (inter-tarefas), e na articulação 
Iniciação esportiva para escolares: os impactos na coordenação e no desempenho motor... Nayanne Dias Araújo - Henrique de Oliveira Castro - Allana Lendary Bernardo Ramos - Tatiane Mazzardo • Gabriella Nelli Monteiro • Layla Maria Campos Aburachid

horizontal, as tarefas propõem a estimulação das habilidades com níveis de dificuldades semelhantes (intra-tarefas).

Os conteúdos tratados foram: capacidades coordenativas, habilidades técnicas, capacidades táticas básicas e pequenos jogos por meio de estruturas funcionais (criando situações de superioridade, inferioridade e igualdade numéricas), além dos jogos para o desenvolvimento da inteligência e criatividade tática (JDICT), conforme constante no Quadro 1.

Quadro 1. Características pedagógicas do IEU+EB aplicados na intervenção de ensino.

\begin{tabular}{|c|c|c|c|c|}
\hline & Conteúdos & $\begin{array}{l}\text { Proposta meto- } \\
\text { dológica }\end{array}$ & $\begin{array}{c}\text { Formas de aprendi- } \\
\text { zagem }\end{array}$ & Domínio da prática \\
\hline 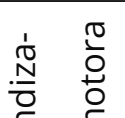 & $\begin{array}{l}\text { Capacidades } \\
\text { coordenativas }\end{array}$ & EB & \multirow{5}{*}{$\begin{array}{l}\text { Incidental } \\
\text { estimulada por am- } \\
\text { bas as propostas } \\
\text { metodológicas }\end{array}$} & \multirow{5}{*}{$\begin{array}{c}\text { Geral } \\
\text { estimulada por am } \\
\text { bas as propostas } \\
\text { metodológicas }\end{array}$} \\
\hline 峁 & $\begin{array}{l}\text { Habilidades técnicas } \\
\text { gerais }\end{array}$ & EB & & \\
\hline \multirow{3}{*}{$\begin{array}{l}\frac{0}{0} \\
\frac{\pi}{0} \\
\frac{\pi}{0} \\
\frac{0}{0} \\
\frac{0}{0} \\
\frac{0}{0} \\
\frac{0}{0} \\
0\end{array}$} & $\begin{array}{c}\text { Capacidades táticas } \\
\text { gerais } \\
\end{array}$ & EB & & \\
\hline & Estruturas funcionais & IEU & & \\
\hline & JDICT & IEU & & \\
\hline
\end{tabular}

Nota: JDICT = Jogos para o desenvolvimento da inteligência e criatividade tática; IEU = Iniciação Esportiva Universal; $E B=$ Escola da bola.

Fonte: autores.

\section{Categorização das aulas}

As informações referentes à categorização das aulas, trazem à tona os temas abordados via conteúdos de ensino da IEU+EB. Por meio da categorização das aulas, apresentam-se as distribuições das frequências de ocorrência e do tempo referente ao indicar tipos de segmento de aula e conteúdos de ensino, conforme a Tabela 1. 
Iniciação esportiva para escolares: os impactos na coordenação e no desempenho motor... Nayanne Dias Araújo - Henrique de Oliveira Castro - Allana Lendary Bernardo Ramos • Tatiane Mazzardo • Gabriella Nelli Monteiro • Layla Maria Campos Aburachid

Tabela 1. Frequência de ocorrência e tempo do indicador tipos de segmento da aula, quantidade de participantes e delimitação espacial.

\begin{tabular}{|l|l|l|l|l|l|l|l|c|}
\hline Tipos segmento de aula & $f$ & Min. $\begin{array}{c}\text { Quantidade } \\
\text { de partici- } \\
\text { pantes }\end{array}$ & $f$ & \multicolumn{1}{|c|}{ Min. } & $\begin{array}{c}\text { Delimitação } \\
\text { espacial }\end{array}$ & $f$ & Min. \\
\hline Conversa com o professor & 85 & 453,28 & 1 a 1 & 13 & 155,70 & Círculo central & 85 & 453,28 \\
\hline JDICT & 12 & 182,70 & Individual & 22 & 298,23 & $\begin{array}{l}\text { Quadra de } \\
\text { vôlei }\end{array}$ & 20 & 266,42 \\
\hline $\begin{array}{l}\text { Capacidades coordenati- } \\
\text { vas }\end{array}$ & 20 & 269,72 & $1 \times 1+1$ & 1 & 7,80 & $\begin{array}{l}1 / 2 \text { quadra de } \\
\text { vôlei }\end{array}$ & 22 & 253,30 \\
\hline $\begin{array}{l}\text { Habilidades técnicas } \\
\text { gerais }\end{array}$ & 18 & 210,33 & 2 a 2 & 1 & 9,13 & $\begin{array}{l}\text { Quadra de } \\
\text { futsal }\end{array}$ & 19 & 246,18 \\
\hline Capacidades táticas gerais & 23 & 274,48 & $2 \times 2$ & 5 & 61,77 & $\begin{array}{l}1 / 2 \text { quadra de } \\
\text { futsal }\end{array}$ & 9 & 130,48 \\
\hline Estruturas funcionais & 12 & 134,07 & $2 \times 2+1$ & 1 & 12,73 & $\begin{array}{l}1 / 4 \text { quadra de } \\
\text { vôlei }\end{array}$ & 11 & 120,20 \\
\hline & & & $2 \times 1$ & 3 & 32,13 & $\begin{array}{l}1 / 4 \text { quadra de } \\
\text { futsal }\end{array}$ & 4 & 54,72 \\
\hline & & & $3 \times 3$ & 1 & 6,32 & & & \\
\hline & & & $3 \times 1$ & 1 & 9,25 & & & \\
\hline & & & $3 \times 3+1$ & 2 & 24,08 & & & \\
\hline
\end{tabular}

Nota: JDICT = Jogos para o desenvolvimento da inteligência e criatividade tática; $f$ = frequência de ocorrência; min = minutos.

Fonte: autores.

A duração média das sessões de aula foi de 90 minutos, totalizando 1.524,58 minutos nas 17 sessões. Para além dos conteúdos transmitidos, inseriu-se a conversa com o professor. Nesse item constam o tempo para a orientação pedagógica, mudança/troca das atividades e hidratação, o que explica seu amplo quantitativo temporal (453,28 min).

Para identificar se o emprego do método de ensino foi aplicado coerentemente, utilizou-se o cálculo do qui-quadrado $\left(x^{2}\right)$, obtendo-se as frequências observadas e esperadas. Os demais tipos de segmento presentes tratam dos conteúdos das propostas metodológicas da IEU+EB. Os subitens JDICT, capacidades táticas gerais e estruturas funcionais são inerentes ao desenvolvimento da capacidade de jogo, que somados representaram 591,25 minutos do 
Iniciação esportiva para escolares: os impactos na coordenação e no desempenho motor...

Nayanne Dias Araújo - Henrique de Oliveira Castro - Allana Lendary Bernardo Ramos - Tatiane Mazzardo • Gabriella Nelli Monteiro • Layla Maria Campos Aburachid

tempo total da intervenção. Verificou-se que o desenvolvimento da capacidade de jogo sobressaiu frente ao demais conteúdos (capacidades coordenativas e habilidades técnicas gerais) voltados ao desenvolvimento da aprendizagem motora $\left(X^{2}=20,635 ; p=0,000\right)$.

Os subitens que compuseram o item Quantidade de participantes (Tabela 1) estão relacionados à disposição dos alunos durante as atividades, realizadas individualmente, em cooperação, em situações de igualdade, superioridade e inferioridade numérica. Encontrou-se diferenças significativas entre as frequências observadas e esperadas para a configuração 3 ou mais $\left(x^{2}=143,341\right.$; $p=0,000$ ), indicando uma prevalência do ensino por meio dos jogos.

A delimitação espacial mostra o espaço em que ocorriam as conversas com o professor, assim como as atividades durante as sessões de aulas. Os espaços da quadra foram divididos em campos de jogo reduzidos, para a aplicação das atividades desenvolvidas em pequenos grupos. Os subitens quadra de voleibol, 1/2 quadra de voleibol e quadra de futsal foram utilizados com mais frequência nas aulas, e condizem com o item número de participantes (Tabela 1), pois as atividades com maior quantidade de alunos envolvidos por grupo requereram um espaço maior. Logo, não se encontrou diferenças significativas entre as frequências observadas e esperadas $\left(x^{2}=0,653 ; p=0,957\right)$, confirmando que, em pelo menos três espaços, as atividades ocorreram de maneira distribuída. O espaço círculo central representou o maior tempo das aulas, pois foi utilizado para o item de tipos de segmento da aula, previamente citado, Conversa com professor, e não entrou no cálculo no qui-quadrado, pois a intenção foi observar as diferenças nos locais das ações práticas.

\section{Análise estatística}

Após a verificação da normalidade dos dados, aplicou-se estatística descritiva (frequência absoluta e relativa, mediana e intervalo interquartílico), inferencial (teste de Wilcoxon, Mann-Witney e 
Iniciação esportiva para escolares: os impactos na coordenação e no desempenho motor... Nayanne Dias Araújo - Henrique de Oliveira Castro - Allana Lendary Bernardo Ramos - Tatiane Mazzardo • Gabriella Nelli Monteiro • Layla Maria Campos Aburachid

aderência) e a equação de probabilidade condicional. Para avaliação da concordância intra e inter-observador, utilizou-se o Kappa Cohen, com avaliações de teste-reteste sendo realizadas com intervalo de sete dias e análise atendendo a $10 \%$ da amostra (duas sessões de aula). Os resultados intra e inter-observador da categorização das aulas resultaram em concordância quase perfeita $(0,98$ e 0,96, respectivamente). Foi adotado o valor de $p \leq 0,05$.

\section{Resultados}

Impactos da coordenação motora e desenvolvimento motor, considerando o efeito tempo e sexo

No grupo experimental, as meninas apresentaram melhora nas tarefas (trave, salto lateral e transposição), os meninos nas tarefas (trave, salto monopedal e transposição), e ambos obtiveram melhoras no quociente motor geral (Tabela 2).

Tabela 2. Coordenação motora considerando os efeitos tempo e sexo para o grupo experimental

\begin{tabular}{|c|c|c|c|c|c|c|}
\hline & Sexo & & Pré-teste & & Pós-teste & $p$ \\
\hline Tarefas & \multirow{6}{*}{ O+ } & $\mathrm{Md}$ & (I IQ - III IQ) & $\mathrm{Md}$ & (I IQ - III IQ) & \\
\hline Trave de equilíbrio & & 87,0 & $(75,0-95,0)$ & 107,0 & $(90,0-111,0)$ & $0,018 *$ \\
\hline Salto monopedal & & 87,0 & $(75,0-96,0)$ & 94,0 & $(76,0-99,0)$ & 0,236 \\
\hline Salto lateral & & 77,0 & $(74,0-110,0)$ & 96,0 & $(81,0-109,0)$ & $0,075^{*}$ \\
\hline Transposição & & 81,0 & $(57,0-89,0)$ & 91,0 & $(86,0-101,0)$ & $0,018^{*}$ \\
\hline Quociente motor & & 103,0 & $(96,0-111,0)$ & 117,0 & $(104,0-124,0)$ & $0,018 *$ \\
\hline Trave de equilíbrio & \multirow{5}{*}{ ro } & 84,0 & $(80,0-99,0)$ & 102,0 & $(95,5-109,5)$ & $0,008^{*}$ \\
\hline Salto monopedal & & 82,0 & $(75,0-92,0)$ & 95,0 & $(81,0-99,0)$ & $0,008^{*}$ \\
\hline Salto lateral & & 92,0 & $(82,5-104,5)$ & 101,0 & $(92,0-110,0)$ & 0,059* \\
\hline Transposição & & 76,0 & $(72,5-83,5)$ & 91,0 & $(62,5-96,5)$ & 0,373 \\
\hline Quociente motor & & 102,0 & $(100,0-122,0)$ & 115,0 & $(105,0-122,5)$ & $0,006^{*}$ \\
\hline
\end{tabular}

No desenvolvimento motor foi possível perceber melhoras estatisticamente significativas para o grupo experimental nos esco- 
Iniciação esportiva para escolares: os impactos na coordenação e no desempenho motor...

Nayanne Dias Araújo - Henrique de Oliveira Castro - Allana Lendary Bernardo Ramos - Tatiane Mazzardo • Gabriella Nelli Monteiro • Layla Maria Campos Aburachid

res de locomoção, controle de objetos e quociente motor (Tabela 3). O grupo controle não obteve melhora significativa após a intervenção em tarefa ou escore algum para as duas variáveis (coordenação motora e desenvolvimento motor).

Tabela 3. Desenvolvimento motor intragrupo considerando os efeitos tempo e sexo para o grupo experimental

\begin{tabular}{|c|c|c|c|c|c|c|}
\hline & Sexo & & ré-teste & & ós-teste & $p$ \\
\hline Escores & \multirow{4}{*}{ O+ } & Md & (I IQ - III IQ) & Md & (I IQ - III IQ) & \\
\hline Locomoção & & 7,0 & $(5,0-8,0)$ & 9,0 & $(9,0-12,0)$ & $0,018 *$ \\
\hline Controle de objetos & & 5,0 & $(3,0-7,0)$ & 8,0 & $(7,0-9,0)$ & $0,018 *$ \\
\hline Quociente motor & & 76,0 & $(67,0-82,0)$ & 94,0 & $(88,0-100,0)$ & $0,018^{*}$ \\
\hline Locomoção & \multirow{3}{*}{ ro } & 7,0 & $(5,5-7,5)$ & 11,0 & $(7,5-13,0)$ & $0,007 *$ \\
\hline Controle de objetos & & 5,0 & $(4,0-6,0)$ & 9,0 & $(7,0-10,0)$ & $0,005^{*}$ \\
\hline Quociente motor & & 73,0 & $(71,5-99,0)$ & 100,0 & $(82,0-104,5)$ & $0,005^{*}$ \\
\hline
\end{tabular}

Nota: $q=$ meninas; $\hat{\delta}=$ meninos; Wilcoxon ${ }^{*} p \leq 0,05$

Fonte: autores.

A Tabela 4 apresenta os resultados de tamanho de efeito, agrupando-se ambos os sexos, para coordenação e para o desenvolvimento motor.

Tabela 4. Tamanho do efeito dos grupos experimental e controle nas comparações intergrupos, considerando o efeito tempo para coordenação e desenvolvimento motor

\begin{tabular}{|l|l|l|l|l|l|l|l|l|l|}
\hline $\begin{array}{c}\text { Coordenação } \\
\text { motora }\end{array}$ & \multicolumn{3}{|c|}{$\Delta$ Controle } & \multicolumn{3}{c|}{$\Delta$ Experimental } & \multicolumn{2}{|l|}{} & \multicolumn{2}{|l|}{} \\
\hline Tarefas & Md & P25 & P75 & Md & P25 & P75 & p & TE & \\
\hline Trave & $11,9 \%$ & $-1,7 \%$ & $26,5 \%$ & $17,3 \%$ & $4,2 \%$ & $29,6 \%$ & 0,208 & - & - \\
Salto monopedal & $19,7 \%$ & $7,9 \%$ & $26,7 \%$ & $5,5 \%$ & $0,0 \%$ & $15,7 \%$ & $0,022^{*}$ & 0,511 & Grande \\
Salto lateral & $30,0 \%$ & $11,2 \%$ & $40,6 \%$ & $6,9 \%$ & $0,0 \%$ & $11,8 \%$ & $0,012^{*}$ & 0,560 & Grande \\
Transposição & $27,7 \%$ & $4,2 \%$ & $40,0 \%$ & $9,4 \%$ & $-1,5 \%$ & $28,5 \%$ & 0,093 & - & - \\
Quociente motor & $14,8 \%$ & $10,3 \%$ & $16,9 \%$ & $8,6 \%$ & $4,9 \%$ & $12,9 \%$ & $0,016 *$ & 0,538 & Grande \\
\hline
\end{tabular}


Iniciação esportiva para escolares: os impactos na coordenação e no desempenho motor... Nayanne Dias Araújo - Henrique de Oliveira Castro - Allana Lendary Bernardo Ramos - Tatiane Mazzardo • Gabriella Nelli Monteiro • Layla Maria Campos Aburachid

\begin{tabular}{|c|c|c|c|c|c|c|c|c|c|}
\hline $\begin{array}{l}\text { Desenvolvimen- } \\
\text { to motor }\end{array}$ & \multicolumn{3}{|c|}{$\Delta$ Controle } & \multicolumn{3}{|c|}{$\Delta$ Experimental } & \multirow[b]{2}{*}{$p$} & \multirow{2}{*}{\multicolumn{2}{|c|}{ TE }} \\
\hline Escores & Md & $\mathrm{P} 25$ & P75 & $\mathrm{Md}$ & P25 & P75 & & & \\
\hline Locomoção & $-20,0 \%$ & $-33,3 \%$ & $20,8 \%$ & $53,6 \%$ & $24,3 \%$ & $85,7 \%$ & 0,001 * & 0,739 & Grande \\
\hline $\begin{array}{l}\text { Controle de ob- } \\
\text { jetos }\end{array}$ & $0,0 \%$ & $-50,0 \%$ & $175,0 \%$ & $62,5 \%$ & $24,3 \%$ & $129,2 \%$ & 0,080 & - & - \\
\hline Quociente motor & $2,3 \%$ & $-12,3 \%$ & $16,8 \%$ & $27,9 \%$ & $14,9 \%$ & $46,2 \%$ & $<0,001 *$ & 0,844 & Grande \\
\hline
\end{tabular}

Nota: $T E=$ Tamanho de efeito ( $r=z-s c o r e / \sqrt{ } n$ - Classificação Cohen), Mann-Whitney *p $\leq 0,05$ Fonte: autores.

Os resultados apontam impactos considerados grandes para a coordenação motora no grupo experimental, com exceção das tarefas trave e transposição do KTK. Também se vê grandes impactos para o mesmo grupo para o escore de locomoção e quociente motor.

\section{Chance de alteração na classificação do nível de coordenação e desenvolvimento motor}

A chance de alteração obtida por meio da equação de probabilidade condicional (Tabela 5), apresenta o número de vezes que o grupo experimental foi superior ou inferior ao grupo controle na evolução da classificação obtida. 
Iniciação esportiva para escolares: os impactos na coordenação e no desempenho motor... Nayanne Dias Araújo • Henrique de Oliveira Castro • Allana Lendary Bernardo Ramos • Tatiane Mazzardo • Gabriella Nelli Monteiro • Layla Maria Campos Aburachid

Tabela 5. Chance de alteração na classificação dos grupos experimental e controle para os níveis de coordenação e desenvolvimento motor.

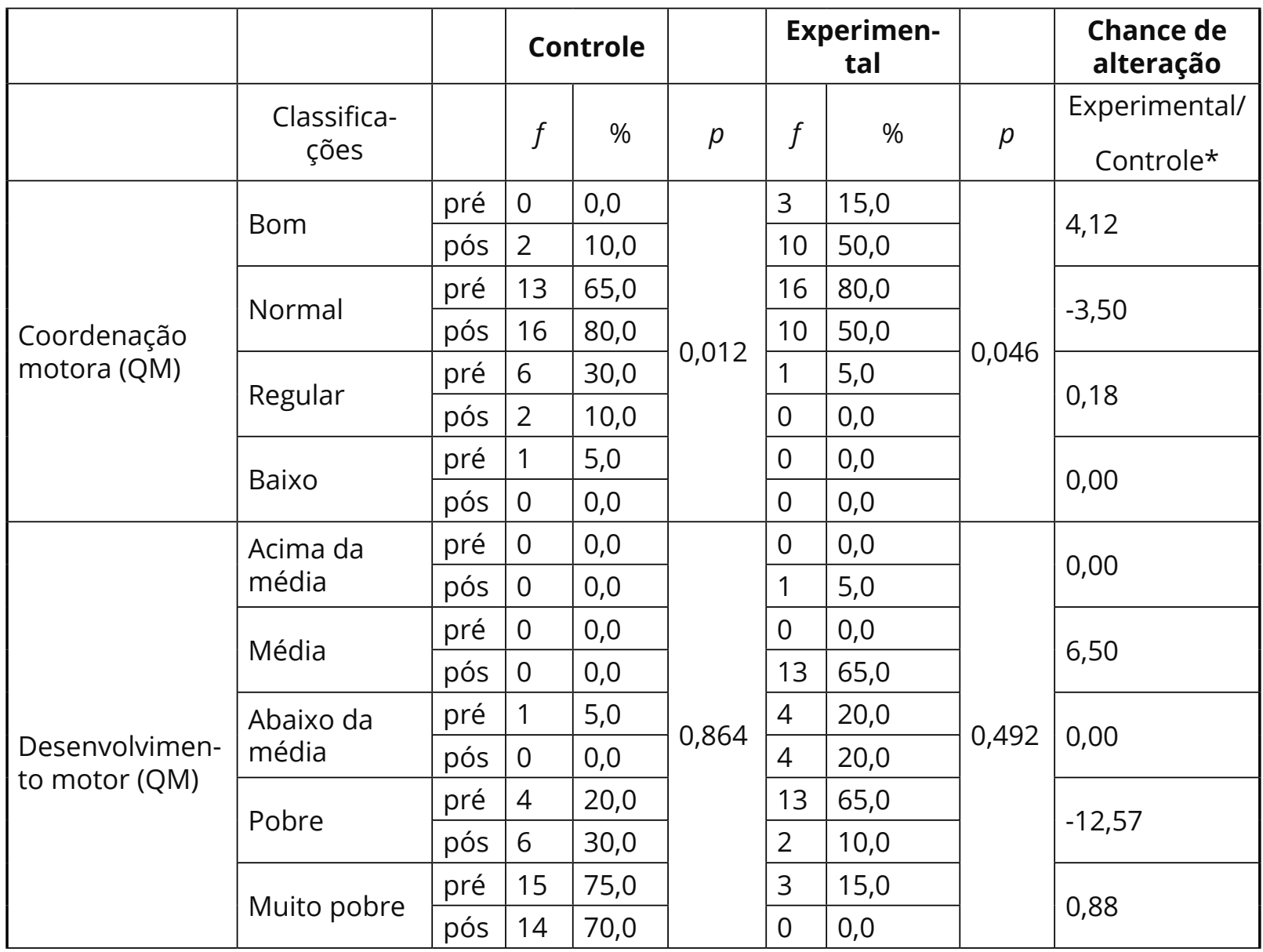

Nota: $Q M=$ quociente motor; $f$ = frequência de ocorrência de participantes; *Chance de Alteração na Classificação (((PÓSG2-PRÉG2) / (NG2-PRÉG2)) / ((PÓSG1-PRÉG1) / (NG1-PRÉG1))). Valores positivos indicam chance para aumentar o $n$ no momento pós, no grupo experimental em relação a chance do grupo controle. Valores negativos indicam chance para reduzir o n no momento pós, no grupo G2 em relação a chance do grupo G1; $p=$ Qui-quadrado para tendência. Fonte: autores.

Quanto ao nível de coordenação motora após a intervenção, o grupo experimental aumentou em 4,12 vezes sua chance de manter seus participantes na classificação da coordenação boa, o que fez, consequentemente, reduzir a chance de mantê-los classificados como coordenação normal (- 3,15 vezes). Ao considerar o nível de desenvolvimento motor, o grupo experimental reduziu em 12,57 vezes a chance de manter seus participantes na classificação pobre, o que por outro lado, aumentou em 6,5 vezes sua chance de se manter na classificação média. 
Iniciação esportiva para escolares: os impactos na coordenação e no desempenho motor...

Nayanne Dias Araújo - Henrique de Oliveira Castro - Allana Lendary Bernardo Ramos - Tatiane Mazzardo • Gabriella Nelli Monteiro • Layla Maria Campos Aburachid

\section{Discussão}

O estudo objetivou identificar os impactos do IEU+EB para a formação esportiva geral de crianças de oito a 10 anos, no contexto de ensino escolar, sobre o nível de coordenação motora e do desenvolvimento motor, considerando os efeitos tempo e sexo, e analisar a chance de alteração na classificação das variáveis. A hipótese do estudo de que a proposta metodológica híbrida do IEU+EB promoveria melhorias na coordenação motora e no desenvolvimento motor das crianças foi confirmada. A categorização das sessões de aula creditou a coerência da aplicação dos conteúdos de ensino, prevalecendo dentre eles o desenvolvimento da capacidade de jogo frente aos conteúdos voltados ao desenvolvimento da aprendizagem motora, confirmando a execução das propostas metodológicas da IEU+EB (GRECO et al., 2020), que levam em consideração que os indivíduos na faixa etária do estudo devem aprender jogando. Os conteúdos de JDICT, capacidades táticas básicas e estruturas funcionais, inerentes ao desenvolvimento da capacidade de jogo, representaram somados 591,25 minutos do tempo total da intervenção. Tais resultados corroboram o estudo com IEU de Lages e colaboradores (2021) que, em sua intervenção, computaram o tempo de 789,12 minutos em 22 aulas de Educação Física. Da mesma forma, com o estudo de Silva (2015), também com IEU que apontou o conteúdo capacidades táticas básicas presentes em $86 \%$ das aulas.

A maior frequência de atividades no estudo se apresentou na configuração de 3 ou mais participantes, seguida de atividades individuais, que caracterizaram a aplicação dos conteúdos de capacidade de jogo e de capacidades coordenativas, respectivamente. Quanto a capacidade de jogo, o estudo de Aburachid e colaboradores (2019), por se tratar de uma intervenção com um método integrativo com 48 jovens do ensino médio $(15,1 \pm 0,64$ anos), também estimulou este conteúdo, mas quatro vezes menos em tempo de aplicação. Isso se deve ao maior foco em jogos reduzidos e voltados à especificidade do badminton, e também por estimular 
Iniciação esportiva para escolares: os impactos na coordenação e no desempenho motor...

Nayanne Dias Araújo - Henrique de Oliveira Castro - Allana Lendary Bernardo Ramos - Tatiane Mazzardo • Gabriella Nelli Monteiro • Layla Maria Campos Aburachid

mais tempo o domínio de prática específica, explorando pouco o uso de espaços grandes da quadra, mas sim, as $1 / 2$ quadras de badminton. Lages e colaboradores (2021), apesar de aplicarem o IEU, concederam aos alunos, em todas as aulas da intervenção, 184,15 minutos de prática de jogo formal de basquetebol (39,63 minutos), futsal ( 96,01 minutos) e handebol (48,51 minutos), o que não ocorreu no presente estudo.

Destaca-se que o presente estudo foi realizado com crianças com faixa etária mais baixa (oito a 10 anos), que devem ser bastante estimuladas quanto aos conteúdos para desenvolvimento da capacidade de jogo (JDICT, capacidades táticas básicas e estruturas funcionais) e a aprendizagem motora (coordenação motora e habilidades técnicas gerias), além de que, esta não se trata de uma fase de aprendizagem de especialização esportiva, mas sim de iniciação. Apesar de Greco, Morales e Aburachid (2017) indicarem que, na faixa etária do presente estudo, as crianças são capazes de realizar atividades de coordenação motora em duplas, esse conteúdo foi tratado de maneira menos complexa, pois os alunos ainda não tinham experiência prática anterior de coordenação motora com bola. Dessa forma, tal conteúdo foi aqui estimulado, em sua maioria, na configuração de participação individual $(298,23$ minutos), para que posteriormente fosse estimulado em duplas (1 a 1) (155,7 minutos). Esta dificuldade é reforçada com os resultados do estudo de Silva, Zampier e Silva (2016), que encontram valores de referência fracos para a coordenação motora com bola em uma amostra de 911 escolares de 7 a 15 anos de idade, de ambos os sexos.

Collet e colaboradores (2007), com o voleibol infantil, aplicaram a maioria de suas atividades na configuração de 3 ou mais alunos, computando maior frequência de ocorrência para o uso da quadra inteira. Porém, não se pode apontar que essa informação corrobora o presente estudo, pois, no estudo de Collet e colaboradores (2007), os jogos reduzidos foram os mais aplicados, e, em muitos casos, utiliza-se neles toda a quadra de voleibol. O mesmo ocorreu no estudo realizado por Mazzardo e colaboradores (2020) 
Iniciação esportiva para escolares: os impactos na coordenação e no desempenho motor...

Nayanne Dias Araújo - Henrique de Oliveira Castro - Allana Lendary Bernardo Ramos - Tatiane Mazzardo • Gabriella Nelli Monteiro • Layla Maria Campos Aburachid

no handebol. Tal fato é bem distinto do ocorrido aqui, uma vez que o uso de grandes espaços serviu para a estimulação da capacidade geral de jogo como: jogos de mão, pé, raquete, bastão; jogos de invasão, de campo, de rede, dentre outros sem especificidade esportiva.

Nas comparações considerando o tempo e sexo para coordenação motora, os estudos apontam que escolares do sexo masculino obtiveram escores médios superiores aos do sexo feminino, mesmo quando não encontradas diferenças estatisticamente significativas entre os mesmos (SILVA; ZAMPIER; SILVA, 2016); FERNANDES; MOURA; SILVA, 2017; PELOZIN et al., 2009; LOPES et al., 2003). Neste estudo, as meninas apresentaram dificuldades na tarefa de salto monopedal, enquanto os meninos no salto lateral; ambas exigiam a capacidade de ritmo, que não foi foco direto das atividades nas sessões de aula, mas inerente a elas. Vaz e colaboradores (2021) e Lages e colaboradores (2021) também encontraram resultados positivos na coordenação motora de escolares, mas sem distinção de sexo.

No desenvolvimento motor, percebeu-se melhoras estatisticamente significativas para o grupo experimental em ambos os sexos e a não melhoria do grupo controle para nenhuma tarefa ou quociente motor. Esse resultado corrobora os encontrados nos estudos de intervenção de Nobre e colaboradores (2012), Costa e colaboradores (2014), Souza e colaboradores (2016) e Nobre, Bandeira e Valentini (2016), que obtiveram melhores resultados para o sexo masculino e feminino para os escores de locomoção e controle de objetos.

Os resultados obtidos pelos impactos da intervenção sobre a coordenação motora foram grandes para o grupo experimental, com exceção das tarefas trave e transposição, assemelhando-se ao estudo de Lages e colaboradores (2021) e de Fernandes, Moura e Silva (2017). Lages e colaboradores (2021), em sua intervenção com IEU, encontraram efeito grande para duas tarefas, e Fernandes, Moura e Silva (2017) constataram um efeito grande nas tarefas salto monopedal, salto lateral, trave e médio na trans- 
Iniciação esportiva para escolares: os impactos na coordenação e no desempenho motor...

Nayanne Dias Araújo - Henrique de Oliveira Castro - Allana Lendary Bernardo Ramos - Tatiane Mazzardo • Gabriella Nelli Monteiro • Layla Maria Campos Aburachid

posição, em 43 escolares com idades entre 6 e 7 anos, de ambos os sexos, participantes de um programa de aulas com abordagem Desenvolvimentista voltadas ao atletismo. Em contrapartida, na intervenção que conteve as capacidades táticas básicas da EB, ocorrida com escolares com idade entre 5 e 10 anos no contraturno, Vaz e colaboradores (2021) encontraram impactos grandes em todas as tarefas do KTK.

Nos impactos para o desenvolvimento motor, os efeitos foram grandes nos escores de locomoção e quociente motor para o grupo experimental. $\mathrm{Na}$ análise intergrupos, também se evidenciou grandes efeitos para o grupo experimental, apresentando diferenças estatisticamente significativas para o escore de locomoção e quociente motor. Esses resultados se aproximam com os do estudo de Nobre, Bandeira e Valentini (2016), em que os efeitos para os escores de locomoção foram grandes, o que não ocorreu aqui com os escores de controle de objetos. Destaca-se que os participantes do estudo não estavam acostumados a utilizar materiais diversificados nas aulas, o que gerou maior dificuldade para na execução das tarefas de controle de objetos.

Finalmente, a análise da chance de alteração na classificação da coordenação motora e do desenvolvimento motor mostrou mudanças temporais do processo, pelos cálculos de razão de chances e razão de prevalência, análise pouco comum nos estudos da educação física, mas sim, na área da epidemiologia, como afirmam Francisco e colaboradores (2008). O estudo de Lopes e colaboradores (2015) encontrou, por meio da razão de chance, que o índice de massa corporal, a circunferência da cintura, o percentual de gordura corporal e a razão cintura/estatura estão positiva e significativamente relacionados à pouca coordenação motora dos sujeitos de ambos os sexos. Neste estudo, pode-se inferir que o grupo que participou da intervenção teve mais chances de manter ou aumentar as classificações de coordenação motora e desenvolvimento motor em relação ao grupo controle. 
Iniciação esportiva para escolares: os impactos na coordenação e no desempenho motor...

Nayanne Dias Araújo - Henrique de Oliveira Castro - Allana Lendary Bernardo Ramos - Tatiane Mazzardo • Gabriella Nelli Monteiro • Layla Maria Campos Aburachid

\section{Conclusão}

Conclui-se que os resultados dos impactos grandes após a intervenção e da chance de alteração da classificação, em sua maioria, reforçam a melhoria dos níveis de coordenação motora e desenvolvimento motor. Apesar de existirem demais estudos de intervenção que avaliaram tais variáveis no país, este foi o primeiro estudo que encontrou resultados efetivos na aplicação dessa proposta híbrida (IEU+ EB), confirmando a efetividade de métodos que exploram a aprendizagem incidental dos alunos.

Como limitação, pelo fato de o espaço de intervenção ser o real ambiente escolar, no horário das aulas de Educação Física, por vezes a quadra ficava ocupada com outras aulas ou ensaios para eventos. Nesses dias, as aulas eram transferidas para um espaço reduzido da quadra ou esperava-se o término dos ensaios para iniciar, diminuindo sua duração.

Recomenda-se a realização de mais estudos de campo que busquem verificar a aplicabilidade de variadas propostas metodológicas sobre a aprendizagem e desenvolvimento motor e conhecimento tático que influenciam no processo de ensino-aprendizagem de escolares. Pretende-se, com esse estudo, contribuir para as investigações de intervenção voltados para o estímulo da aprendizagem incidental, confirmando seu aporte empírico para o aprendizado dos alunos na fase de iniciação esportiva, tanto na escola quanto em clubes e escolinhas de esportes.

\section{Referências}

ABURACHID, L.M.C.; SILVA, S.R.; CLARO, J.N.; GRECO, P.J. O nível de coordenação motora após um programa de treino em futsal. Revista Brasileira de Futsal e Futebol, São Paulo, v. 7, n. 23, p. 25-34, 2015. 
Iniciação esportiva para escolares: os impactos na coordenação e no desempenho motor... Nayanne Dias Araújo - Henrique de Oliveira Castro - Allana Lendary Bernardo Ramos • Tatiane Mazzardo • Gabriella Nelli Monteiro • Layla Maria Campos Aburachid

ABURACHID, L.M.C.; SILVA, S.R.; ARAÚJO, N.D.; GRECO, P.J. Badminton: possibilidades de ensino aplicadas ao contexto da educação física escolar. Journal of Physical Education, Maringá, v. 30, n. 1, e-3055, 2019.

BETTEGA, O.B.; GALATTI, L.R.; GODOY, S.I.; MEDINA, A.A.; SCAGLIA, A.J. Proceso de enseñanza-entrenamiento de jóvenes en el fútbol: posibilidades a partir de un modelo ondulatorio. SPORT TK -

Revista EuroAmericana de Ciencias del Deporte, v. 8, n. 2, p. 17-25, 2019.

BRAUNER, L.M.; VALENTINI, N.C. Análise do desempenho motor de crianças participantes de um programa de atividades físicas. Journal of Physical Education, v. 20, p. 205-216, 2009.

CABRAL, F.A.; ABURACHID, L.M.C.; GRECO, P.J. Futsal feminino universitário: análise de uma proposta metodológica para desenvolvimento das habilidades técnicas. Revista Mineira de Educação Física, Viçosa, Edição Especial, n. 1, p. 1451-1456, 2012.

COLLET, C., NASCIMENTO, J.V.; RAMOS, M.H.K.P.; DONEGÁ, A.L. Processo de Ensino-Aprendizagem-Treinamento no voleibol infantil masculino em Santa Catarina. Revista de Educação Física/ UEM. Maringá, v. 18, n. 2, p. 147-159, 2007.

COSTA, C.L.A.; NOBRE, G.C.; NOBRE, F.S.S.; VALENTINI, N.C. Efeito de um programa de intervenção motora sobre o desenvolvimento motor de crianças em situação de risco social na região do Cariri - CE. Journal of Physical Education, v. 25, n. 3, p. 353-364, 2014.

FERNANDES, S.P.; MOURA, S.S.; SILVA, S.A. Coordenação motora de escolares do ensino fundamental: influência de um programa de intervenção. Journal of Physical Education, Maringá, v. 28, e2842, 2017.

FRANCISCO, P.M.S.B.; DONALISIO, M.R.; BARROS, M.B.A.; CESAR, C.L.G.; CARANDINA, L.; GOLDBAUM, M. Medidas de associação em estudo transversal com delineamento complexo: razão de chances e razão de prevalência. Revista Brasileira de Epidemiologia, São Paulo, v. 11, n. 3, p. 347-355, 2008. 
Iniciação esportiva para escolares: os impactos na coordenação e no desempenho motor... Nayanne Dias Araújo - Henrique de Oliveira Castro - Allana Lendary Bernardo Ramos • Tatiane Mazzardo • Gabriella Nelli Monteiro • Layla Maria Campos Aburachid

GRECO, P.J.; BENDA, R.N. Iniciação esportiva universal: da aprendizagem motora ao treinamento técnico. v.1. Belo Horizonte: Editora UFMG, 1998.

GRECO, P.J.; MEMMERT, D.; MORALES, J.C.P. The Effects of Deliberate Play on Tactical Performance in Basketball. Perceptual and Motor Skills, v. 110, n. 3, p. 849-856, 2010.

GRECO, P.J.; MORALES, J.C.P; ABURACHID, L.M.C. Metodologia do ensino dos esportes coletivos: iniciação esportiva universal, aprendizado incidental - ensino intencional. In: GALATTI, L.R.; SCAGLIA, A.J.; MONTAGNER, P.C.; PAES, R.R. (orgs).

Desenvolvimento de treinadores e atletas: pedagogia do esporte - v. 1. Campinas, SP: Editora da Unicamp, 2017.

GRECO, P.J.; MORALES, J.C.P.; ABURACHID, L.M.C.; LOPES, M.C.; SILVA, S.R.; BENDA, R.N. Iniciação Esportiva Universal: o jogo do "ABC" na alfabetização esportiva. In: LEMOS, K.L.M.; GRECO, P.J.; MORALES, J.C.P. (Org.). 5. Congresso Internacional dos Jogos Desportivos. Belo Horizonte: EEFFTO/UFMG, 2015. p. 335-359. GRECO, P.J.; PRAÇA, G.M.; MORALES, J.C.P.; ABURACHID, L.M.C.; SILVA, S.R. Vinte anos de iniciação esportiva universal: o conceito de jogar para aprender e aprender jogando, um pedagógico ABC-D. In: BOULLOSA, D.; LARA, L.; ATHAYDE, P. (orgs). Treinamento esportivo: um olhar multidisciplinar. Natal, RN: EDUFRN, 174 p, 2020. p. 43-64.

GUPTA, S.K. Intention-to-treat concept: A review. Perspective in Clinical Research, v. 2, n. 3, p. 109-112, 2011.

GURVITCH, R.; METZLER, M. Aligning Learning Activities with Instructional Models. Journal of Physical Education, Recreation \& Dance, v. 84, n. 3, p. 30-37, 2013.

KIPHARD, E. J.; SCHILLING, V. F. Köperkoordinationstest Für Kinder KFK: manual Von Fridhelm Schilling. Weinheim: Beltz Test Gmbh, 1974.

KLEYNEN, M.; BRAUN, S.M.; BLEIJLEVENS, M.H.; LEXIS, M.A.; RASQUIN, S.M.; HALFENS, J.; WILSON, M.R.; BEURSKENS, A.J.; 
Iniciação esportiva para escolares: os impactos na coordenação e no desempenho motor... Nayanne Dias Araújo - Henrique de Oliveira Castro - Allana Lendary Bernardo Ramos - Tatiane Mazzardo • Gabriella Nelli Monteiro • Layla Maria Campos Aburachid

MASTERS, R.S.W. Using a Delphi Technique to Seek Consensus Regarding Definitions, Descriptions and Classification of Terms Related to Implicit and Explicit Forms of Motor Learning. PLoS One, v. 9, n. 4, e100227, 2014.

KRAHENBÜHL, T.; LEONARDO, L. O ensino do sistema defensivo individual no handebol e suas considerações para a iniciação esportiva. Pensar a Prática, v. 21, n. 1, p. 194-206, 2018.

KRÖGER, C.; ROTH, K. Escola da bola: um ABC para iniciantes nos jogos esportivos. São Paulo: Phorte, 2005.

LAGES, E.R.A.; GRECO, P.J.; PRAÇA, G.M.; MOREIRA, P.E.D.; DUARTE, M.G.; MORALES, J.C.P. Ensino-aprendizagem incidental e seus efeitos sobre o conhecimento tático processual e a coordenação motora com bola. Journal of Physical Education, Maringá, v. 32, e3262, 2021.

LOPES, V.P.; MAIA, J.; SILVA, R.; MORAIS, F. Estudo do nível de desenvolvimento da coordenação motora da população escolar (6 a10 anos de idade) da Região Autónoma dos Açores. Revista Portuguesa de Ciência do Desporto, v. 3, n. 1, p. 47-60, 2003. LOPES, L.; SANTOS, R.; MOREIRA, C.; PEREIRA, B.; LOPES, V.P. Sensibilidade e especificidade de diferentes medidas de adiposidade para diferenciação entre pouca/ampla coordenação motora. Jornal de Pediatria, Porto Alegre, v. 91, n. 1, p. 44-51, 2015. MAZZARDO, T.; RIBAS, S.; MONTEIRO, G.N.; SILVA, W.J.B.; ARAÚJO, N.D.; ABURACHID, L.M.C. Programa de ensino no handebol por meio da compreensão e seu efeito no desempenho tático-técnico de crianças. Journal of Physical Education, Maringá, v. 31, e3169, 2020.

MEMMERT, D. Developing creative thinking in a gifted sport enrichment program and the crucial role of attention processes. High Ability Studies, v. 17, n. 1, p. 101-115, 2006.

MEMMERT, D.; ROTH, K. The effects of non-specific and specific concepts on tactical creativity in team ball sports. Journal of Sports Sciences, v. 25, n. 12, p. 1423-1432, 2007. 
Iniciação esportiva para escolares: os impactos na coordenação e no desempenho motor... Nayanne Dias Araújo - Henrique de Oliveira Castro - Allana Lendary Bernardo Ramos - Tatiane Mazzardo • Gabriella Nelli Monteiro • Layla Maria Campos Aburachid

MESQUITA, I.; GRAÇA, A.; GOMES, A.R.; CRUZ, C. Examining the impact of a step game approach to teaching volleyball on student tactical decision making and skill execution during game play. Journal of Human Movement Studies, v. 48, p. 469-492, 2005. MOREIRA J.P.A.; LOPES, M.C.; MIRANDA-JÚNIOR, M.V.; VALENTINI, N.C.; LAGE, G.M.; ALBUQUERQUE, M.R. Körperkoordinationstest Für Kinder (KTK) for Brazilian Children and Adolescents: Factor Analysis, Invariance and Factor Score. Frontiers in Psychology, v. 19, n.10, p. 2524, 2019.

MOREIRA, V.J.P.; MATIAS, C.J.A.S.; GRECO, P.J. A influência dos métodos de ensino-aprendizagem-treinamento no conhecimento tático processual no futsal. Motriz: Revista de Educação Física, Rio Claro, v. 19, n. 1, p. 84-98, 2013.

NOBRE, S.S.; BANDEIRA, P.F.R.; LIMA, M.S.C.; NOBRE, G.C. Intervenção motora como fator determinante no desenvolvimento motor: estudo comparativo e quase experimental. Revista Acta Brasileira do Movimento Humano, v. 2, n. 2, p. 76-85, 2012.

NOBRE, S.S.; BANDEIRA, P.F.R; VALENTINI, N.C. Atrasos motores em crianças desfavorecidas socioeconomicamente: um olhar Bioecológico. Motricidade, v. 12, n. 2, p. 59-69, 2016.

PELOZIN, F.; FOLLE, A.; COLLET, C.; BOTTI, M.; NASCIMENTO, J.V. Nível de coordenação motora de escolares de 09 a 11 anos da rede estadual de ensino da cidade de Florianópolis/SC. Revista Mackenzie de Educação Física e Esporte, v. 8, n. 2, p.123-132, 2009.

PÍFFERO, C.M.; VALENTINI, N.C. Habilidades especializadas do tênis: um estudo de intervenção na iniciação esportiva com crianças escolares. Revista Brasileira de Educação Física e Esporte, São Paulo, v. 24, n. 2, p. 149-63, 2010.

RAAB, M. SMART-ER: a situation model of anticipated response consequences in tactical decisions in skill acquisition-extended and revised. Frontiers in Psychology, v. 5, p. 1-5, 2015. 
Iniciação esportiva para escolares: os impactos na coordenação e no desempenho motor... Nayanne Dias Araújo - Henrique de Oliveira Castro - Allana Lendary Bernardo Ramos - Tatiane Mazzardo • Gabriella Nelli Monteiro • Layla Maria Campos Aburachid

RINK, J. Teaching Physical Education for Learning. (6nd Ed.) ST. Louis: Times Mosby College Publishing, 2010.

SILVA, S.A. Ensino dos jogos esportivos na Educação Física escolar: o desenvolvimento da capacidade de jogo. Revista Brasileira de Ciência e Movimento, v. 23, n. 1, p. 95-102, 2015.

SILVA, S.A.; ZAMPIER, J.E.L.C.; SILVA, F.L. Valores de referência da coordenação com bola. Revista Acta Brasileira do Movimento Humano, v. 6, n. 3, p. 40-51, 2016

SOARES, V.O.V.; SANTOS, H.F.S.; LIMA, M.R.M.; ABURACHID, L.M.C; GRECO, P.J. Validação de protocolo de categorização de metodologias de ensino nos esportes coletivos com base na Iniciação Esportiva Universal - uma escola da bola: exemplo do futebol. EF Desportes Revista Digital, Buenos Aires, año 15, n. 144, 2010. SOUZA, F.C.S.; BANDEIRA, P.F.R.; VELENTINI, N.C.; RAMALHO, M.H.S.; CARVALHAL, M.I.M. Impacto de um programa social esportivo nas habilidades motoras de crianças de 7 a 10 anos de idade. Motricidade, v. 12, supl. 1, p. 69-75, 2016.

ULRICH, D.A. Test of gross motor development-2. Austin: TX: PRO-ED, 2000.

VALENTINI, N.C.; BARBOSA, M.L.L.; CINI, G.V.; PICK, R.K.; SPESSATO, B.C.; BALBINOTTI, M.A.A. Teste de desenvolvimento motor grosso: validade e consistência interna para uma população gaúcha. Revista Brasileira de Cineantropometria \& Desempenho Humano, v. 10, n. 4, p. 399-404, 2008.

VAZ, M.C.S.O.; CAMPOS, W.; KERKOSKI, M.J.; AFONSO, G. Análise dos efeitos de um programa de intervenção baseado na estrutura TARGET e "Escola da Bola" na coordenação motora grossa. Caderno de Educação Física e Esporte, v. 19, n. 1, p. 19-24, 2021.

VICKERS, J. Instructional Design for Teaching Physical

Education. Champaign IL: Human Kinetics. 1990. 
Iniciação esportiva para escolares: os impactos na coordenação e no desempenho motor...

Nayanne Dias Araújo - Henrique de Oliveira Castro - Allana Lendary Bernardo Ramos - Tatiane Mazzardo • Gabriella Nelli Monteiro • Layla Maria Campos Aburachid

\section{Publisher}

Universidade Federal de Goiás. Faculdade de Educação Física e Dança. Publicação no Portal de Periódicos UFG. As ideias expressadas neste artigo são de responsabilidade de seus autores, não representando, necessariamente, a opinião dos editores ou da universidade. 